\title{
Population response to environmental change: A model with an alternate stable state
}

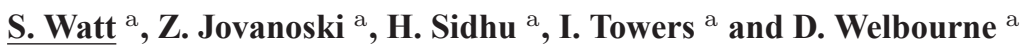 \\ ${ }^{a}$ School of Physical, Environmental and Mathematical Sciences, UNSW Canberra, ACT 2600. \\ Email: $\underline{\text { simon.watt@adfa.edu.au }}$
}

\begin{abstract}
In ecology, the theory of alternative stable states predicts that ecosystems can exist under multiple "states". Ecosystems may transition from one stable state to another, in what is known as a state shift. Typically such shifts are considered as instantaneous and isolated non-interacting events resulting from environmental shocks, whose dynamics resemble the dynamics of a ball in a "potential well". More often however, ecological systems are subject to continuous variation due to environmental drivers such as rainfall, temperature, among others, and these interact with the ecosystem dynamics to alter the potential well. Such drivers are known to impact intra-species interactions.

We explore a single species population model with logistic-like growth and a variable crowding parameter that measures the strength of the intra-species interaction. The crowding parameter is treated as a state-variable whose dynamics is described via a potential function subject to external environmental drivers.
\end{abstract}

Keywords: Population dynamics, alternative stable state 


\section{INTRODUCTION}

In many ecological systems, their dynamics fluctuate around some stable long-term trend or average. Despite variations due to the seasonality of the vegetation and reproductive cycles, the long-term behaviour does not vary widely. However it is occasionally observed that small changes in the external factors can lead to a fundamental change in the long-term behaviour of the system and another equilibrium state is found. The existence of alternative stable states has become an influential concept in ecology (Holling 1973, May 1977). An example given in Scheffer \& Carpenter (2003) was the vegetation in the Sahara region. After a long period of around three and a half thousand years of a slow decline in the vegetation cover, there was a sudden shift in the amount of oceanic sedimentation and the Sahara region was changed to a desert.

This sudden change of state in the ecological system is analogous to the trajectory of a ball rolling on a surface with hills and valleys - a surface potential (Beisner et al. 2003). The changes in the system arise due to small perturbations in system parameters. These perturbations cause the ecological system to move across threshold boundaries that separate different basins of attraction. The long term behaviour of the system is dictated by local minima of the potential surface. For sufficiently small perturbations of system parameters, the long-term behaviour will be centered around some long-term average. However as the perturbations are increased, the system could be attracted to another stable state. We will explore a simple model which exhibits this types of behaviour.

\section{PROPOSED MODEL}

Let us begin by assuming a model that incorporates logistic-like growth

$$
\frac{d N}{d t}=r N-b N^{2}
$$

where $N$ is the population size. The first term represents the natural increase in the population with intrinsic growth rate $r$ in an environment with unlimited resources. The second term represents the effect of intraspecies competition due to finite resources. The parameter $b$ measures the strength of that interaction. One interpretation of $b$ is that of a crowding factor: when resources are abundant $b$ is small, and when resources are depleted $b$ increase thus intensifying competition. When $r$ and $b$ are constant, there exist two steady states: an unstable state with zero population $(N=0)$ and a stable state that is the result of the balance between growth and crowding - the population reaches a stable equilibrium at the system's carrying-capacity $K=r / b$.

To extend this model, we consider the crowding factor $b$ as a state variable that is described through a differential equation. In addition, to model a system which mimics the alternative stable state hypothesis, we propose a potential function which schematically is given in Figure 1. For different environmental conditions, we expect that the system could be in one or the other of the stable states corresponding to the minima of the potential function, and which occur at crowding factor $b_{1}$ or $b_{2}$. This in turn leads to two very different carrying-capacities $K_{1}$ and $K_{2}$, respectively.

We assume that the potential function is symmetric about the average of $b_{1}$ and $b_{2}\left(b_{a v}=\left(b_{1}+b_{2}\right) / 2\right)$. One
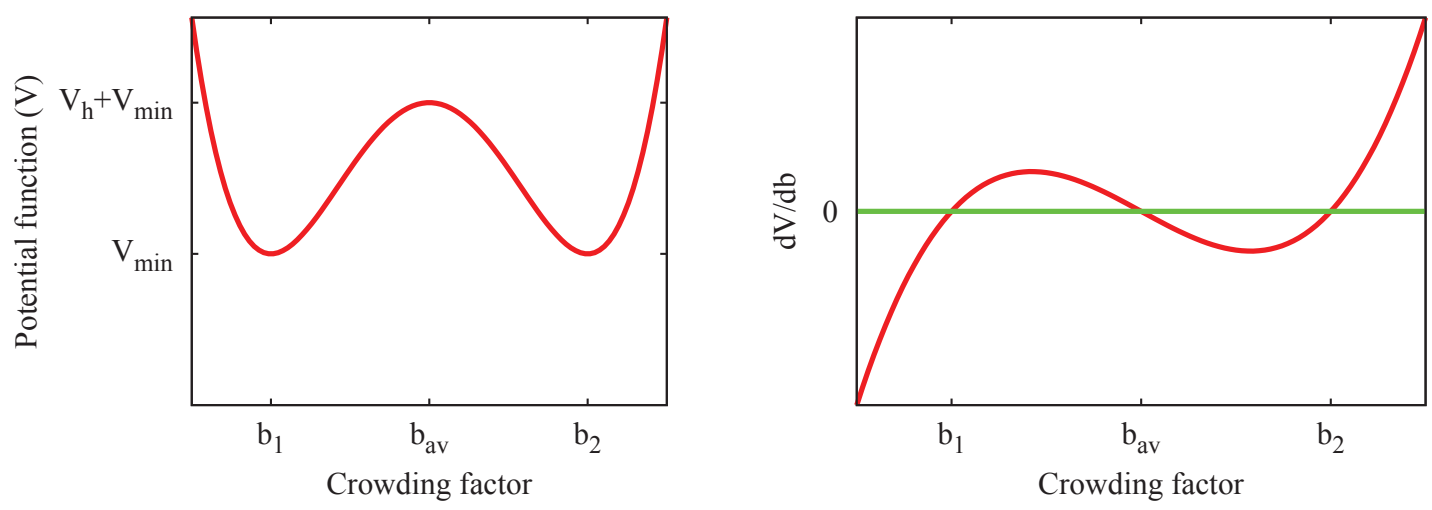

Figure 1. Schematic of the potential function and its derivative. 


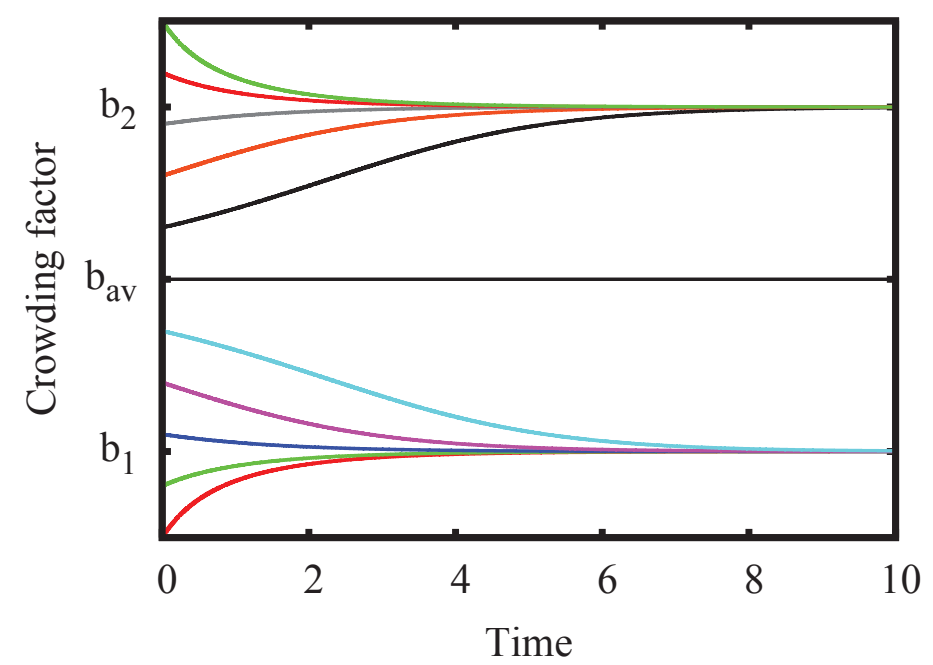

Figure 2. The crowding factor without any forcing for various initial conditions.

of the simplest forms the potential function can take is a quartic function of the form

$$
V(b)=\frac{16\left(V_{a v}-V_{\min }\right)}{\left(b_{1}-b_{2}\right)^{2}}\left(b-b_{1}\right)^{2}\left(b-b_{2}\right)^{2}+V_{\text {min }},
$$

where $V_{\min }$ and $V_{a v}$ are the value of the potential function at $b=b_{i}(i=1,2)$ and $b=b_{a v}$ respectively.

The extra equation to model the changing crowding factor is the over-damped differential equation (Srinivasan \& Kumar 2015)

$$
\frac{d b}{d t}=-\frac{d V}{d b}
$$

One of the features to note about this system is that the evolution of $b$ is decoupled from the population. In Figure 2 we can see that the system quickly evolves away from the unstable state $\left(b_{a v}\right)$ to one of the two stable states $\left(b_{1}\right.$ and $\left.b_{2}\right)$. This means that for any given initial environmental conditions the population settles to one of the two stable states. The effect of external perturbations is explored next.

\subsection{Periodic forcing}

The system (1) and (2) possesses two stable equilibria (and one unstable equilibrium point). However, we are interested in the case where there is a transition from one to the other. To explore this we incorporate a forcing term to drive $b$. The resulting system equations are

$$
\begin{aligned}
\frac{d N}{d t} & =r N-b N^{2}, \\
\frac{d b}{d t} & =-\frac{d V}{d b}+f(t),
\end{aligned}
$$

where $f(t)$ is a forcing term which could represent environmental drivers, such as seasonality. For simplicity we assume

$$
f(t)=\epsilon \cos \left(\frac{2 \pi t}{T}\right),
$$

where $T$ is the period of forcing and $\epsilon$ is the forcing amplitude. (If $t$ is measured in years then setting $T=1$ represents annual forcing: here we consider an arbitrary time unit.)

Suppose that $r$ is fixed and that the population is initially in equilibrium with it environment with parameter $b_{1}$. For the case $\epsilon=0$, the population remains in equilibrium with its surrounding environment, $N(t)=K_{1}$, 
see Figure 3. For $\epsilon=0.02$, a small fluctuation about the mean of $b_{1}$ give rise to fluctuations in the population about $K_{1}$. Increasing to $\epsilon=0.04$ increases the size of fluctuations in the population about $K_{1}$, but remains locally bound. However, increasing the amplitude of the forcing term beyond some critical amplitude $\epsilon_{c r}$ causes a transition from $b_{1}$ to the large crowding factor $b_{2}$, and back again, exhibiting an oscillatory behaviour with a fixed period. The corresponding population initially declines, due to increased competition, followed by an increase in the population when the environment is driven to a more favourable state.

(a) $\epsilon=0$
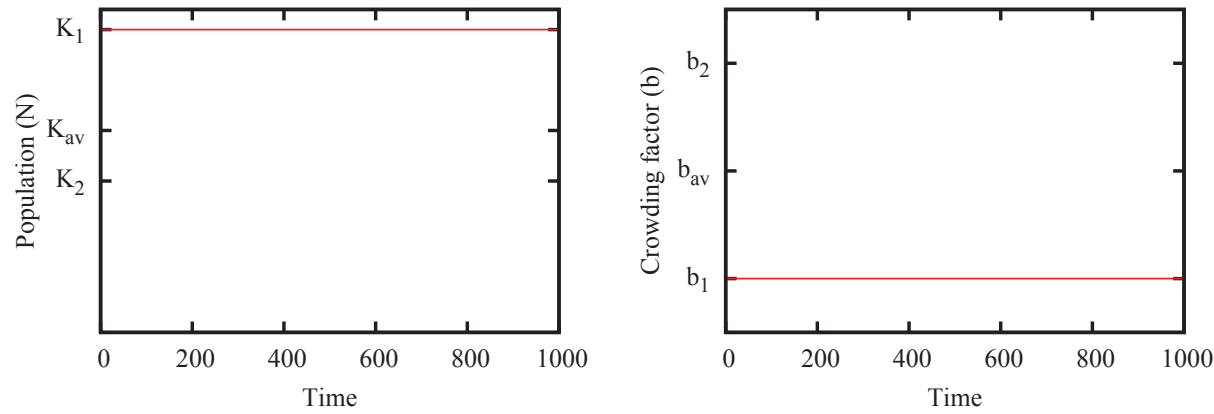

(b) $\epsilon=0.02$
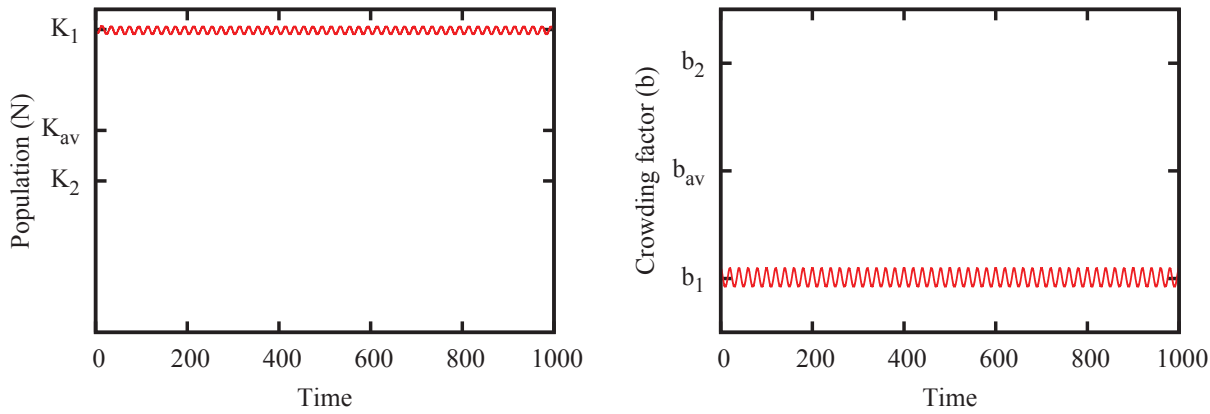

(c) $\epsilon=0.04$
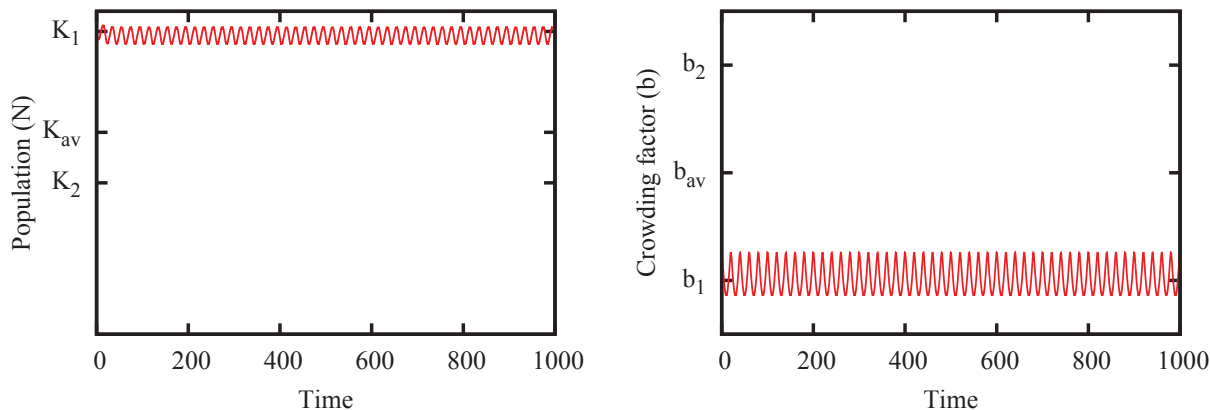

(d) $\epsilon=0.05$
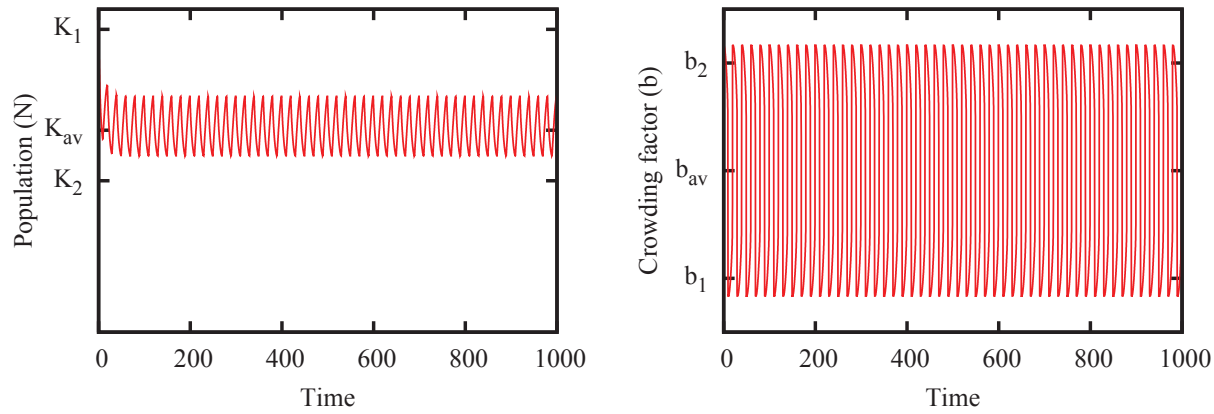

Figure 3. The evolution of the population and crowding factor as the strength of the forcing $(\epsilon)$ is increased. Here $T=20$. 
Three key points are evident from Figure 3. Firstly, the relative amplitude of the oscillations in the population are smaller than those in the crowding factor. This is due to the nonlinearity in the equation describing the population. Secondly, although the crowding factors $b_{1}$ and $b_{2}$ are reached repeatedly, the population does not return to either $K_{1}$ nor $K_{2}$. This is dependent on the value of $r$. For larger $r$, the population is more responsive to the changes in the state of the environment via changes in $b$. Thirdly, we note that $b_{a v}$ is located mid-way between $b_{1}$ and $b_{2}$, the populations oscillates about $K_{a v}$ which is asymmetrically located between $K_{1}$ and $K_{2}$. This means that once the population decreases towards $K_{2}$ it becomes harder to force the population to a larger value. This has consequences for the conservation of such ecosystems (Dudgeon et al. 2010).

\subsection{Impulsive forcing}

An alternative approach to transitioning between alternative states is to include an impulse to the rate equation for $b$. Such a scenario could represent a one-off weather event, such a fire on a land ecosystem (Schowalter 2012) or the effects of a environmental contamination on a marine ecosystem (Knowlton 2004). This has the effect of "kicking" the system from one stable state to the other.

The system may be written as

$$
\begin{aligned}
\frac{d N}{d t} & =r N-b N^{2} \\
\frac{d b}{d t} & =-\frac{d V}{d b}+f(t)+I(t),
\end{aligned}
$$

where $I(t)$ is the impulsive component of an external perturbation. As an illustration, we use a scaled Gaussian to approximate a delta function

$$
I(t)=\left(b_{2}-b_{1}\right) \frac{1}{\sqrt{2 \pi} \sigma} \exp \left(-\frac{\left(t-t_{i m p}\right)^{2}}{2 \sigma^{2}}\right) .
$$

This function is the delta function in the limit $\sigma \rightarrow 0$. The population dynamics for this scenario is depicted in Figure 4 where $t_{i m p}=500$ and $\sigma=0.1$. Here we have assumed that we are transitioning from the higher population (with $b_{1}$ ) to lower population (with $b_{2}$ ) stable state. Even if $\epsilon<\epsilon_{c r}$, the impact of the impulse it to switch the dynamics irreversibly to the other stable state.
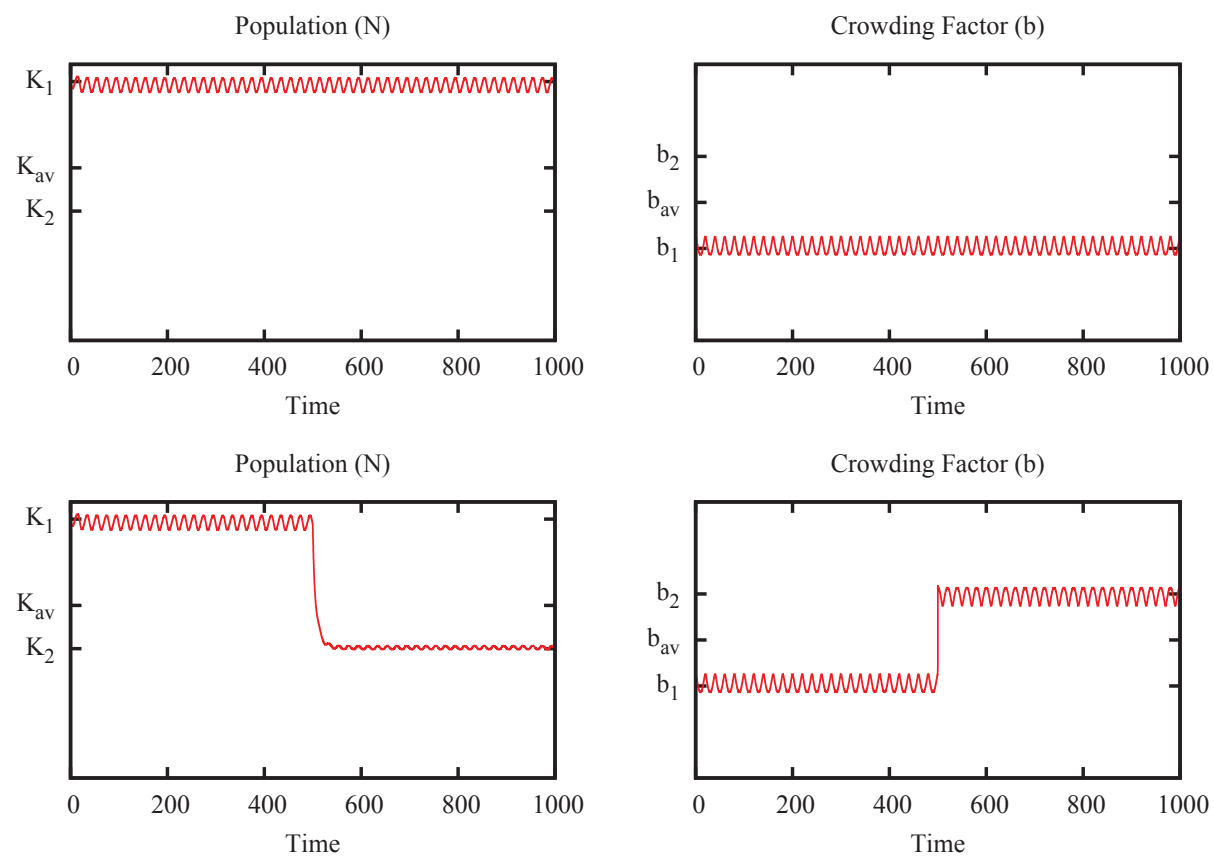

Figure 4. The solutions in the upper panels have a periodic forcing and the solutions in the lower panels have a periodic forcing plus an impulse force at $t=500$. 


\section{CONCLUSIONS}

In this paper we have considered an ecosystem which possesses the behaviour of transitioning between two alternative stable states. The ecosystem describes a single population that is subject to environmental drivers that affect the intra-species competition for resources via the so-called crowding factor. By modelling the crowding factor as a state variable we introduce a potential function approach to describe the induced changes due to environmental perturbations.

This enables us to describe how environmental drivers can cause the system to switch between two stable states. We also considered a scenario that includes a one-off kick to the system that forces the system to move from one basin of attraction to another.

The work presented here is a preliminary study into the behaviour of a simple population model exhibiting alternate stable states. Obvious extensions is to include stochastic drivers of $b$ (or an other system parameters) (Anderson et al. 2015, 2016).

Ultimately, our hope is to investigate whether a "potential well" approach can increase our understanding of ecosystem dynamics, especially whether such an approach can better inform conservation practices. Conservationists may aim to modify the potential in such a way as to eliminate the less favourable state and force the population to a different, more favourable state, one for which the population size is larger. For instance, the practice of quarantining large areas to fishing helps to increase the population of fish stocks by eliminating one state in favour of an other (Ghosh et al. 2017).

\section{REFERENCES}

Anderson, C., Jovanoski, Z., Sidhu, H. S. \& Towers, I. N. (2016), 'Logistic equation with a simple stochastic carrying capacity', ANZIAM Journal 56, C431-C445.

Anderson, C., Jovanoski, Z., Towers, I. N., \& Sidhu, H. S. (2015), A simple population model with a stochastic carrying capacity, in T. Weber, M. McPhee \& R. Anderssen, eds, 'MODSIM2015, 21st International Congress on Modelling and Simulation. Modelling and Simulation Society of Australia and New Zealand, December 2015', pp. 36-42. ISBN: 978-09872143-5-5. www.mssanz.org.au/modsim2015/A1/anderson.pdf.

Beisner, B. E., Haydon, D. T. \& Cuddington, K. (2003), 'Alternative stable states in ecology', Frontiers in Ecology and the Environment 1, 376-382.

Dudgeon, S. R., Aronson, R. B., Bruno, J. F., \& Precht, W. F. (2010), 'Phase shifts and stable states on coral reefs', Marine Ecology Progress Series 413, 201-216.

Ghosh, B., Debprasad, P., Kar, T. K. \& Valverde, J. C. (2017), 'Biological conservation through marine protected areas in the presence of alternative stable states', Mathematical Biosciences 286, 49-57.

Holling, C. S. (1973), 'Resilience and stability of ecological systems', Annual Reviews of Ecological Systems $4,1-23$.

Knowlton, N. (2004), 'Multiple "stable" states and the conservation of marine ecosystems', Progress in Oceanography 60, 387-396.

May, M. R. (1977), 'Thresholds and breakpoints in ecosystems with a multiplicity of stable states', Nature 269, 471-477.

Scheffer, M. \& Carpenter, S. R. (2003), 'Catastrophic regime shifts in ecosystems: linking theory to observation', Tends in Ecology and Evolution 18, 648-656.

Schowalter, T. D. (2012), 'Landscape-level disturbance', Annual Review of Entomology 57, 1-20.

Srinivasan, V. \& Kumar, P. (2015), 'Emergent and divergent resilience behavior in catastrophic shift systems', Ecological Modelling 298, 87-105. 